OPEN ACCESS

Edited by:

Sumitra Deb,

Virginia Commonwealth University,

United States

Reviewed by:

Vijay Menon,

Yale University, United States

Prashant Vijay Thakkar,

Cornell University, United States

Amrita Sule,

Yale University, United States

${ }^{*}$ Correspondence:

Jihui Jia

jiajihui@sdu.edu.cn

Specialty section:

This article was submitted to

Molecular and Cellular Oncology,

a section of the journal

Frontiers in Oncology

Received: 12 August 2021 Accepted: 07 October 2021

Published: 28 October 2021

Citation:

Zheng L, Wu Y, Shen L, Liang $X$, Yang Z, Li S, Li T, Shang $W$, Shao $W$, Wang $Y$, Liu F, Ma L and Jia J (2021) Mechanisms of JARID1B

Up-Regulation and Its Role in

Helicobacter pylori-Induced

Gastric Carcinogenesis.

Front. Oncol. 11:757497. doi: 10.3389/fonc.2021.757497

\section{Mechanisms of JARID1B Up-Regulation and Its Role in Helicobacter pylori-Induced Gastric Carcinogenesis}

\author{
Lixin Zheng ${ }^{1}$, Yujiao $W u^{1}$, Li Shen ${ }^{1}$, Xiuming Liang ${ }^{1}$, Zongcheng Yang ${ }^{1}$, Shuyan $L^{1}{ }^{1}$, \\ Tongyu $L^{1}{ }^{1}$, Wenjing Shang ${ }^{1}$, Wei Shao ${ }^{1}$, Yue Wang ${ }^{1}$, Fen Liu ${ }^{1}$, Lin Ma ${ }^{1}$ and Jihui Jia ${ }^{1,2^{\star}}$ \\ ${ }^{1}$ Key Laboratory of Experimental Teratology, School of Basic Medical Sciences, Shandong University, Jinan, China, \\ 2 Shandong Provincial Key Laboratory of Infection and Immunology, School of Basic Medical Sciences, Shandong University, \\ Jinan, China
}

Gastric cancer (GC) is the third leading cause of cancer-related death worldwide. Helicobacter pylori infection can induce GC through a serial cascade of events, with emerging evidence suggesting the important role of epigenetic alterations in the development and progression of the disease. Here, we report on mechanisms responsible for Jumonji AT-rich interactive domain1B (JARID1B) upregulation in GC and its role in the malignant transformation induced by $H$. pylori infection. We found that upregulation of JARID1B was associated with poorer prognosis, greater tumor purity, and less immune cell infiltration into the tumor. Mechanistically, we showed that the upregulation of JARID1B in human GC was attributed to JARID1B amplification and its induction by $H$. pylori infection. Furthermore, we identified miR-29c as a negative regulator of JARID1B in GC. H. pylori caused downregulation of miR-29c in human GC and thereby contributed to JARID1B upregulation through relieving posttranscriptional regulation. Functionally, we showed that knockdown of JARID1B reduced GC cell proliferation induced by $H$. pylori infection. Subsequently, cyclinD1 (CCND1), a key molecule in GC, was shown to be a target gene of JARID1B. In conclusion, these results suggest that JARID1B may be an oncogene upregulated in human GC and could represent a novel therapeutic target to prevent malignant transformation induced by $\mathrm{H}$. pylori infection.

Keywords: gastric cancer, Helicobacter pylori, JARID1B, miR-29c, carcinogenesis

\section{INTRODUCTION}

Gastric cancer (GC) is one of the most common malignancies worldwide, with $>950,000$ newly diagnosed cases each year (1). Due to the lack of early diagnostic screening, most patients are diagnosed at an advanced stage (2). Therefore, studying the etiology of GC is of great significance for identifying means of early detection and/or prevention.

Gastric carcinogenesis is a complex, multistep, and multifactorial event. Helicobacter pylori infection has long been recognized as a Class I oncogenic factor in GC. H. pylori can cause chronic gastritis, intestinal metaplasia, and even dysplasia, which can be considered precancerous lesions. 
Hence, eradication of $H$. pylori infection can reduce the risk of GC $(3,4)$. Cytotoxin-associated gene A (CagA) is the main virulence factor of $H$. pylori that could be injected into host cells by type IV secretory system (5). As a pathogenic factor, $H$. pylori can induce host genomic instability, including abnormal DNA methylation and disorders of micro-RNA expression (6-8). However, the exact carcinogenic mechanisms responsible for GC induced by $H$. pylori have not yet been fully elucidated.

Growing evidence is suggesting that epigenetic modifications, including histone demethylation, play important roles in the development of human tumors (9). It has been reported that histone demethylases such as those of the KDM1 [lysine (K) demethylase 1] family and Jumonji C (JmjC) domain-containing family are related to carcinogenesis (10). For example, lysine specific demethylase 1 (LSD1) is abnormally expressed in breast cancer, lung cancer, colon cancer, and GC and is involved in the regulation of tumor development (10-12). Our group has also reported that JMJD2B promotes GC cell proliferation and metastasis through cooperation with $\beta$-catenin $(13,14)$. JARID1B (also termed KDM5B) is a member of the JMJC histone demethylase family and was shown to be involved in tumor progression and metastasis, including GC (15-20). However, the mechanism of JARID1B upregulation in GC remains unclear.

Given the possible pathogenetic mechanisms of GC, some of the histone demethylases induced by $H$. pylori infection might contribute to its tumorigenesis and progression $(13,21,22)$. Our group has reported that $H$. pylori can promote the development of GC by enhancing the expression of JMJD2B (13). Overexpression of the $H$. pylori-induced histone demethylase PHF8 exacerbated metastasis and progression of GC via regulation of vimentin (23). However, the role of JARID1B in the malignant transformation induced by $H$. pylori is unclear, and the underlying mechanisms remain to be elucidated. In the present study, we evaluated the clinical significance of JARID1B overexpression. For the first time, we investigated the expression, functions, and molecular mechanisms of JARID1B for H. pyloriinduced GC progression in a series of in vitro and in vivo assays.

\section{MATERIALS AND METHODS}

\section{Cell Culture}

The human gastric adenocarcinoma GES-1, BGC-823, MGC801, SGC-7901, and HGC-27 cell lines were cultured in RPMI1640 medium (Gibco, Carlsbad, CA, USA) supplemented with $10 \%$ fetal bovine serum (FBS), except for AGS in F12 medium (HyClone, USA) supplemented with 10\% FBS (Gibco).

\section{Clinical Samples}

Fourteen RNA samples were harvested from the GC patients undergoing surgical resection in Shandong Tumor Hospital. Atrophic gastritis (AG) specimens were from 63 patients with or without $H$. pylori infection of Jinan Central Hospital. The 13C urea breath test was used to detect the $H$. pylori. Histological typing of the tumors was used to confirm the diagnosis.

\section{H. pylori Cultures and H. pylori- Infected Mice}

Three H. pylori strains were cultured in Brucella broth containing $5 \% \mathrm{FBS}$ at $37^{\circ} \mathrm{C}$ for $48 \mathrm{~h}$ under microaerophilic conditions. The bacteria were added to cells with the multiplicity of infection (MOI) at 100 . C57BL/6 mice (male) were separated into three groups. Among them, group 1 is the control group. Groups 2 and 3 were first given the N-methyl-Nnitrosourea (MNU, $30 \mathrm{ppm}$ ) in the drinking water; 10 weeks later, they were given distilled water for another 2 weeks. Thereafter, Group 3 was given the SS1 strain $\left(1 \times 10^{9}\right.$ colonyforming units (CFU)/ml) every other day for three times. Group 1 was provided with distilled water without MNU or SS1. Finally, all mice were sacrificed after 50 weeks.

\section{Transfection}

JARID1B siRNAs (Sigma-Aldrich, USA), miR-29c mimic, and inhibitor (Ribobio, Guangzhou, China) transfection were performed with the Lipofectamine 2000 (Invitrogen, Carlsbad, CA, USA). Sequences for these siRNAs: 5'-CAUAUUCUCUC UUAGAGGUdTdT-3' (JARID1B sil) and 5' -CAGUGAAUGA GCUCCGGCAdTdT-3' (JARID1B si2).

\section{Colony Formation Assay}

Cells (600 cells/well) were seeded into the plates after respective treatments, then they were incubated for about 8-14 days until colonies appeared. The colonies were then treated with methanol and Giemsa. The experiments were performed three times.

\section{EdU Staining}

DNA synthesis was assessed by the Cell-Light EdU (5-ethynyl-2'deoxyuridine) Apollo488 In Vitro Imaging Kit according to the manufacturer's instructions (RiboBio).

\section{Immunohistochemistry}

Paraffin-embedded sections acquired from samples were deparaffinized and rehydrated. Then, the citric acid buffer was used for antigen retrieval. Next, after $\mathrm{H}_{2} \mathrm{O}_{2}$ treatment and nonspecific antigen blocking for $30 \mathrm{~min}$, sections were incubated with the primary JARID1B antibody (1:100, ab181089, Abcam, Cambridge, UK). Next day, the colorimetric detection was performed using DAB (3,3'-diaminobenzidine) staining kit (Vector Laboratories, USA).

\section{RNA Extraction and RT-PCR}

Total RNA was extracted with TRIzol reagent (Invitrogen, Carlsbad, CA, USA) as per the manufacturer's instructions. RNAs were then reverse-transcribed by the PrimeScript RT reagent Kit with gDNA Eraser (Takara, Japan). Finally, SYBRGreen (TaKaRa, Japan) was then used for the real-time PCR analysis. The primers used in this real-time quantitative PCR (RT-qPCR) assay are as follows: 5'-TGTCACAGTGGA ATATGGAGCTGAC- $3^{\prime}$ and $5^{\prime}$-GCCACTATCAAGATA CTCCTCTTCC-3' for JARID1B; 5'-ATGGAACACCAGCTC CTGTG-3' and 5'-ACCTCCAGCATCCAGGTGGC-3' for CCND 1; 5' -AGTTGCGTTACACCCTTTCTTG-3' and 
5'-CACCTTCACCGTTCCAGTTTT-3' for $\beta$-actin; 5'-GAATT GCTATGTGTCTGGGT-3' and 5'-CATCTTCAAACC TCCATGATG-3' for $\beta 2-\mathrm{M}$.

\section{Western Blotting}

Cells were lysed with the protein lysis buffer supplemented with protease inhibitors for $30 \mathrm{~min}$ on ice to extract the protein. Next, proteins were subjected through sodium dodecyl sulfatepolyacrylamide gel electrophoresis (SDS-PAGE) and transferred to the polyvinylidene fluoride (PVDF) membranes. Then, 5\% nonfat milk was used to block the membranes. The specific primary antibodies and the respective secondary antibodies were also used for incubation. Immunoblots were visualized with ECL (Enhanced chemiluminescent) reagent (Millipore). The primary antibodies were specific for JARID1B (Abcam), $\beta$-actin (Sigma-Aldrich, St. Louis, MO, USA), CCND1 (Cell Signaling, Danvers, MA, USA), and CagA (Santa Cruz Biotechnology, Santa Cruz, CA, USA).

\section{Luciferase Reporter Assay}

The human JARID1B 3'-untranslated region (UTR) and corresponding mutants were cloned into the pMIR-GLO basic luciferase reporter vector (Promega, Madison, WI, USA). GC cells were transfected with the internal control vector pRL-TK and the promoter or $3^{\prime}$-UTR reporters using Roche Transfection Reagent (Roche, Basel, Switzerland) $24 \mathrm{~h}$ after seeding. Luciferase reporter activity was measured by a Luciferase Assay System (Promega) as per the manufacturer's instructions $48 \mathrm{~h}$ after transfection.

\section{Chromatin Immunoprecipitation Assay}

For chromatin immunoprecipitation (ChIP) assay, the SimpleChIP ${ }^{\circledR}$ Enzymatic Chromatin IP Kit (Cell Signaling, Danvers, MA, USA) was used according to the manufacturer's protocol. The precipitated DNA samples were detected with PCR method.

\section{Identification of Upregulated Differentially Expressed Genes}

The original files (.CEL files) and platform files of the GSE54129 dataset in the Gene Expression Omnibus (GEO) database (http:// www.ncbi.nlm.nih.gov/geo/) were downloaded. The raw data files were analyzed using the Robust Multi-array Average algorithm with the "affy" package and the "impute" package in $\mathrm{R} /$ Bioconductor software (version 3.5.3). For the upregulated differentially expressed genes (DEGs), we used the "limma" package and set $\mathrm{P}<0.05$. The inside-software plugin cytoHubba was used to identify hub genes among the genes of interest. This program provides a user-friendly interface to explore important nodes in biological networks and computes using 11 methods (24). Metascape is a free online resource with an automated metaanalysis tool for understanding the biological significance of a large number of genes and can identify enriched pathways and be used for the construction of protein-protein interaction networks from lists of gene and protein identifiers (25).

\section{Statistical Analysis}

All data are presented as means \pm standard deviations from three independent assays. The Student's t-test or Mann-Whitney U test was used to determine the significance of differences in each two-group comparison. Statistical analysis was performed using SPSS version 23.0. Correlation analysis of the data was performed using linear regression. Differences were considered statistically significant when $\mathrm{P}<0.05$.

\section{RESULTS}

\section{Increased JARID1B Expression Predicts Poorer Clinical Outcome in Gastric Cancer Patients}

To identify potential roles of histone demethylases in human GC pathogenesis, we first undertook logistic regression analysis of 18 histone demethylase genes to establish hazard ratio (HR) values for their influence on GC prognosis using data from The Cancer Genome Atlas (TCGA) database. This approach showed that JARID1B and KDM4A (Lysine-specific demethylase 4A) were statistically significant (Figure 1A). Further analysis indicated that only JARID1B had a significant prognostic value in GC patients (Figures 1B and S1). Additionally, we performed a bioinformatics analysis of JARID1B expression profiles from 876 GC patients using the Kaplan-Meier method and found that high expression of JARID1B was significantly correlated with poorer overall survival, first progression survival, and postprogression survival of GC patients (Figures 1C-E). Furthermore, we also carried out an analysis of the GEO databases (e.g., GSE27342 and GSE63089), which showed that JARID1B expression was upregulated in GC tissues (Figures 1F, G). Analysis of 14 GC biopsies and their corresponding adjacent non-tumor tissues confirmed this result (Figure 1H). Similarly, JARID1B was also differentially expressed in several gastric cell lines, including GES-1, AGS, BGC-823, HGC-27, MGC-803, and SGC-7901 (Figure S2). Taken together, the data imply an oncogenic role for histone demethylase JARID1B in GC.

\section{High Expression of JARID1B Is Associated With Greater Tumor Purity and Less Immune Cell Infiltration in Gastric Cancer}

The tumor microenvironment consists of tumor cells, stromal cells, and infiltrating immune cells, and it has been proposed that these variables are valuable for diagnostic and prognostic assessment of tumors. We therefore utilized TIMER (Tumor IMmune Estimation Resource) to assess the role of JARID1B in GC by adopting the ESTIMATE algorithm (from https:// bioinformatics.mdanderson.org/estimate/). This analysis showed that the level of JARID1B messenger RNA (mRNA) was significantly higher in the low immune score and low stromal score group (Figures 2A, B). Furthermore, high expression of JARID1B was statistically significantly associated with immune score (low, $\mathrm{P}<0.0001$ ) and stromal score (low, $\mathrm{P}=$ 0.017 ; Figures 2A, B). The expression of JARID1B was 
A

Logistic regression analysis in TCGA: normal vs tumor

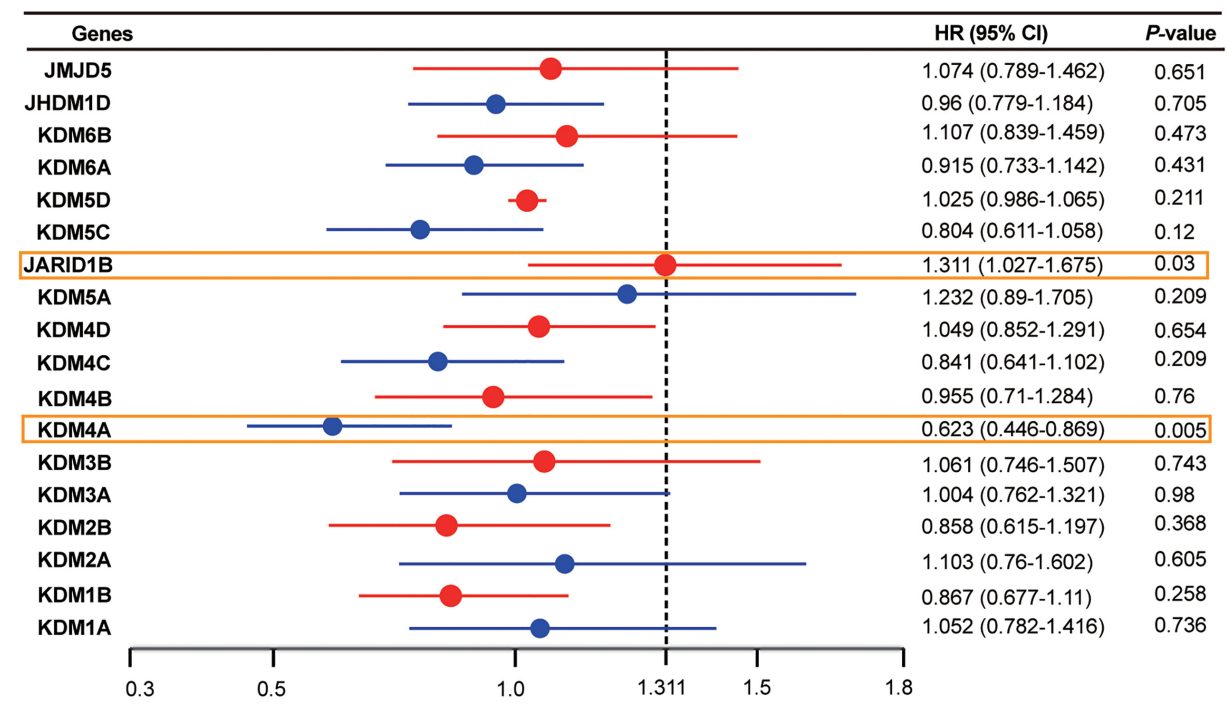

B

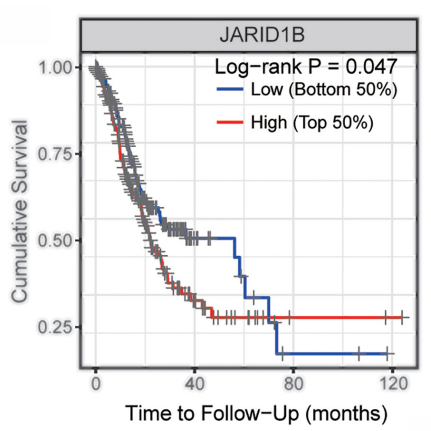

C

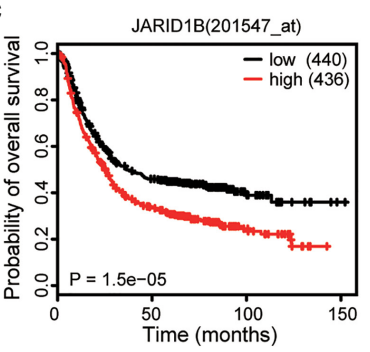

$\mathbf{F}$

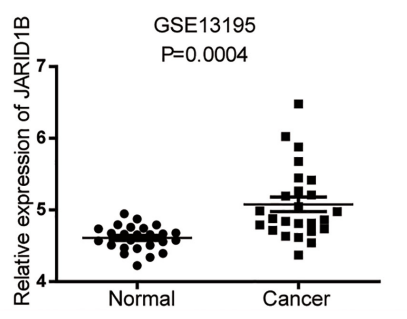

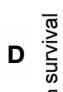

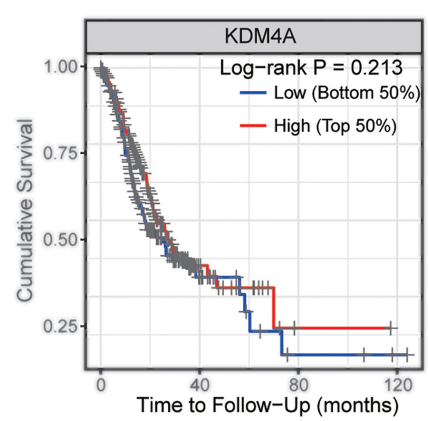

E

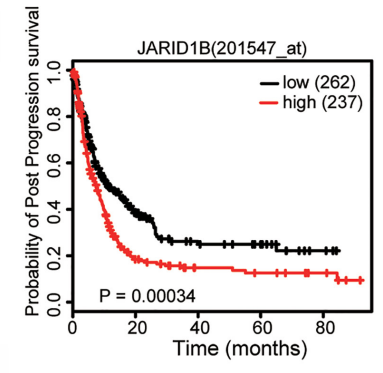

G
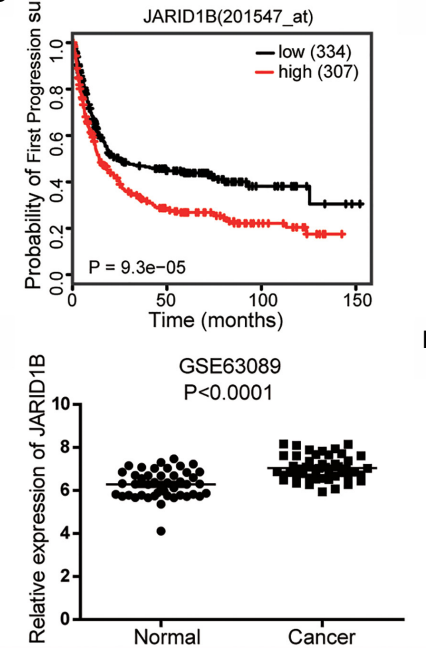

H

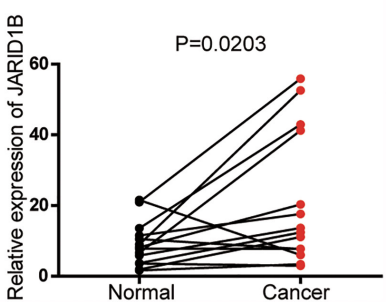

FIGURE 1 | JARID1B overexpression in gastric cancer (GC) patients is associated with poorer prognosis. (A) The logistic regression analysis of 18 histone demethylase gene for the prognosis of GC in The Cancer Genome Atlas (TCGA) database. (B) Further analysis of the prognostic significance of JARID1B and KDM4A in GC patients. (C-E) Relationship between JARID1B expression and overall survival (C), first progression survival (D), the post progression survival (E) in GC patients (log-rank test; $n=876$ ), both data and $P$ values are from the Kaplan-Meier Plotter database. (F, G) GEO RNA sequencing database analysis of the relatively differential expression levels of JARID1B in human GC and paired adjacent normal tissues; the data were derived from GSE13195 (F) and GSE63089 (G). (H) The messenger RNA (mRNA) levels of JARID1B in 14 GC and paired adjacent normal tissues were measured by real-time PCR $(n=14)$. 
A

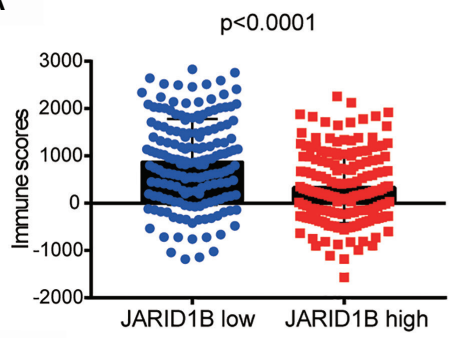

B

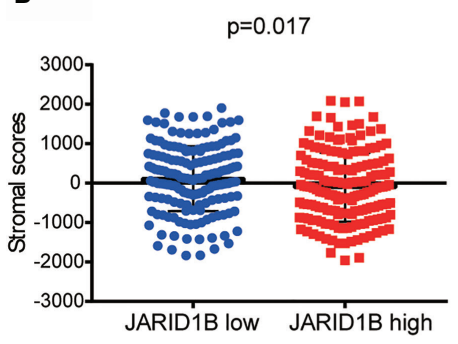

C
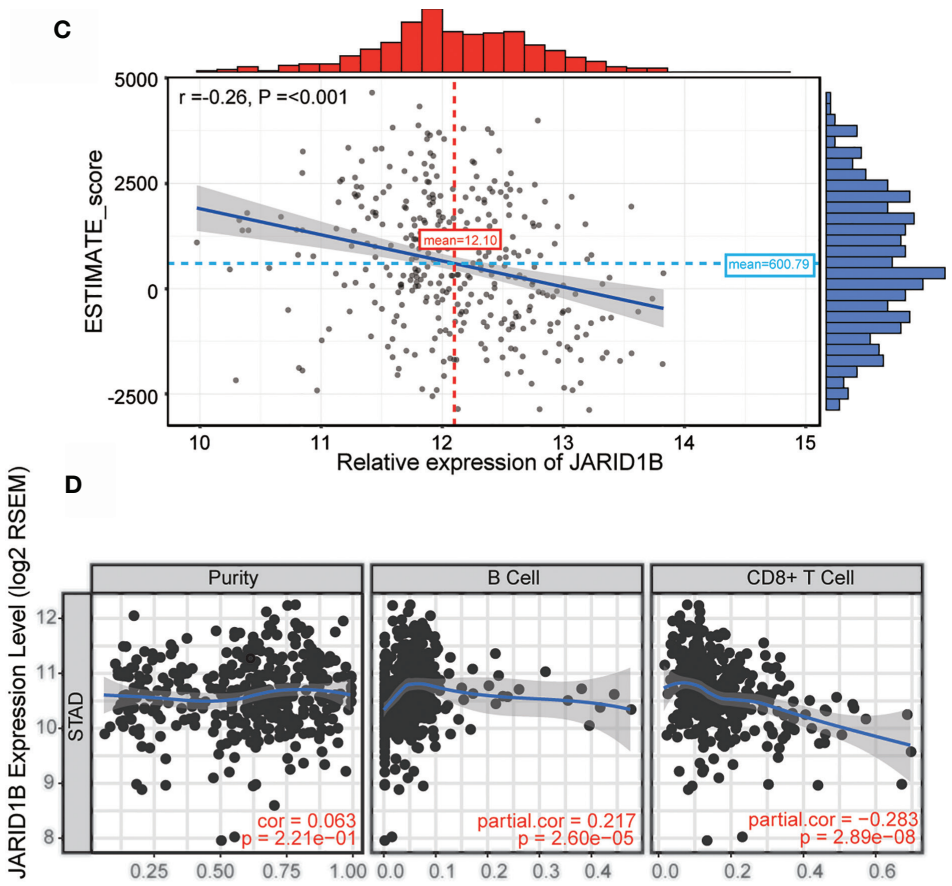

Infiltration Level
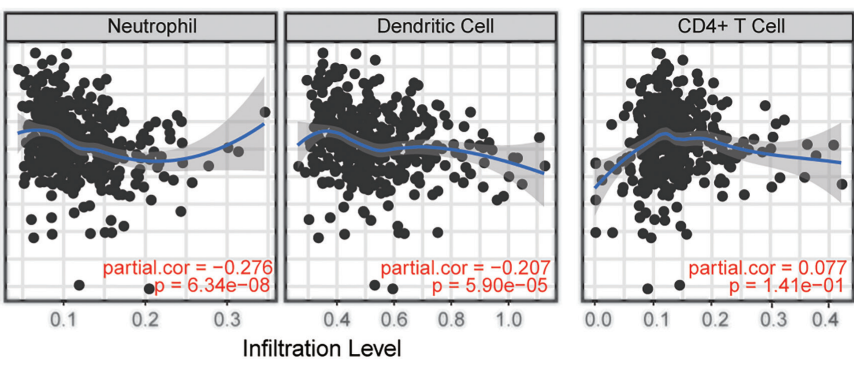

FIGURE 2 | JARID1B overexpression is associated with greater tumor purity and immune infiltration of gastric cancer (GC). (A, B) Relationship between JARID1B expression and the immune score, stromal score group in TIMER of GC. (C) Correlation between JARID1B expression and ESTIMATE score in TIMER of GC. (D) Correlation between JARID1B expression and CD8+ T cells, neutrophils, as well as dendritic cells in TIMER of GC.

negatively correlated with the ESTIMATE score $(\mathrm{r}=-0.26$, $\mathrm{P}<0.001$; Figure 2C), suggesting that JARID1B was positively associated with tumor purity of GC. Moreover, analysis of tumor-infiltrating immune cells showed that high expression of JARID1B was negatively associated with the amount of CD8+ T cells, neutrophils, and dendritic cells in the tumor (Figure 2D).

\section{Gene Amplification of JARID1B in Gastric Cancer}

Next, we investigated the mechanism responsible for JARID1B upregulation in GC. We searched for information on JARID1B gene mutations, as well as the inherent connection between gene copy number and mRNA expression in the "Stomach Adenocarcinoma (TCGA, Provisional)" dataset via the cBioportal program (http://www.cbioportal.org). Notably, gene amplification of JARID1B was frequently found in TCGA GC tissues, and the copy number significantly correlated with levels of JARID1B mRNA expression (Figures 3A, B). This suggests that copy number variation may underlie JARID1B amplification in GC.

\section{H. pylori Promotes the Expression of JARID1B}

H. pylori infection is a risk factor for GC. Hence, we investigated whether $H$. pylori infection influences the abnormal expression of JARID1B in GC. We first analyzed JARID1B expression in H. pylori-infected gastritis tissue by RT-qPCR and documented its significant upregulation relative to $H$. pylori-positive gastritis tissues (Figure 4A). To further investigate the regulation of JARID1B expression by $H$. pylori infection, we used different H. pylori strains (H.p11637 and H.p26695) to infect GC cells (AGS and BGC-823) at different time points. RT-qPCR showed that $H$. pylori infection can upregulate the expression of JARID1B mRNA at different time points (Figures 4B-E). 
A

JARID1B $2 \%|||||||| \mid$

$\operatorname{TCGA}(G C)$

Genetic Alteration Amplification Deep Deletion No alterations
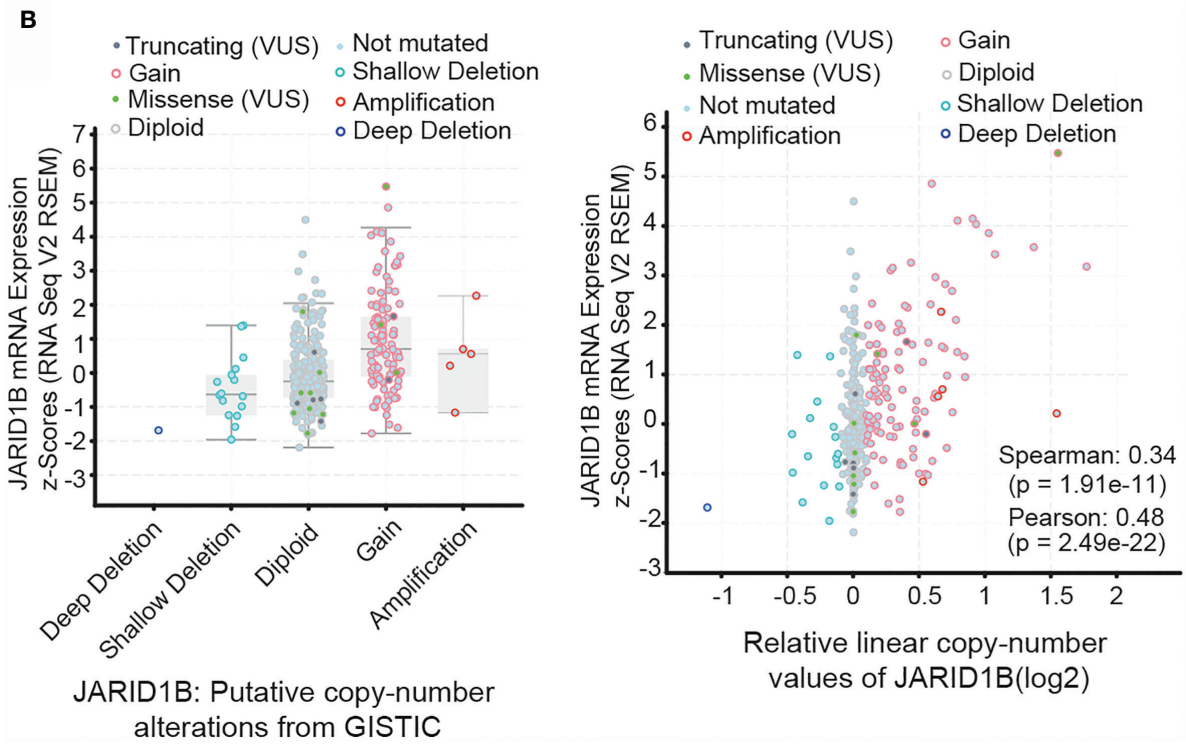

FIGURE 3 | Gene amplification of JARID1B in gastric cancer (GC). (A) cBioPortal (http://www.cbioportal.org/) analysis of the JARID1B genetic amplification in The Cancer Gene Atlas (TCGA) GC tissues. (B) Correlation between JARID1B copy number alteration and the messenger RNA (mRNA) expression.

Furthermore, Western blotting confirmed the upregulation of JARID1B in H. pylori-infected GC cells at the protein level (Figures 4F, G and S3).

H. pylori gavage can promote the progression of precancerous lesions in murine gastric tissue (26). To further investigate the regulation of JARID1B expression by $H$. pylori infection in vivo, we constructed MNU-treated mice with $H$. pylori infection. Immunohistochemistry (IHC) showed that JARID1B was slightly upregulated in the low-dose MNU group compared with the normal gastric mucosa in the control group but was significantly upregulated when low-dose MNU was combined with $H$. pylori infection (Figure $\mathbf{4 H}$ ). These findings indicate that $H$. pylori promotes the expression of JARID1B both in vivo and in vitro.

\section{H. pylori Upregulates the Expression of JARID1B Through the Downregulation of $\mathrm{miR}-29 \mathrm{c}$}

We further explored the mechanisms by which $H$. pylori regulates the expression of JARID1B, focusing on miRNAs, which are important posttranscriptional regulators. Abnormal expression of multiple miRNAs is a known result of $H$. pylori infection $(27,28)$. To investigate the potential role of miRNAs in the regulation of JARID1B, we first screened candidates using TargetScan and found that miR-29c, miR-29b, and miR-29a can bind to the $3^{\prime}$-UTR of JARID1B (Figure 5A). Further analysis of the GEO database (GSE51306) revealed that the expression of miR-29c was downregulated in gastric epithelial cells infected with $H$. pylori, while the expression of miR-29a and miR-29b was not significantly affected (Figures 5B-D). Furthermore, RTqPCR showed that miR-29c expression in H. pylori-infected gastritis tissues was significantly lower than that in uninfected tissues (Figure 5E). In addition, miR-29c expression was downregulated in $H$. pylori-infected AGS and BGC-823 cell lines (Figure 5F).

To determine whether miR-29c regulates JARID1B expression, we transfected these cell lines with miR-29c analogs (mimics) and inhibitors (Figure 5G). Western blotting confirmed that the former decreased the amount of JARID1B protein, while the latter increased it (Figures $\mathbf{5 H}$ and S4). RTqPCR showed that transfection of AGS and BGC-823 cells with miR-29c analogs significantly inhibited JARID1B mRNA expression (Figure 5I). To further investigate whether JARID1B is a direct target of miR-29c, we constructed a fluorescent reporter plasmid containing the JRID1B $3^{\prime}$-UTR of the miR-29c binding site [JARID1B-wild type (WT)-3'-UTR] and a miR-29c binding site-mutated reporter plasmid (JARID1B-Mut- $3^{\prime}$-UTR) (Figure 5J). We found that the miR29c analogs with JARID1B-WT- $3^{\prime}$-UTR reduced the luciferase activity, which was not the case for JARID1B-Mut-3'-UTR 
A

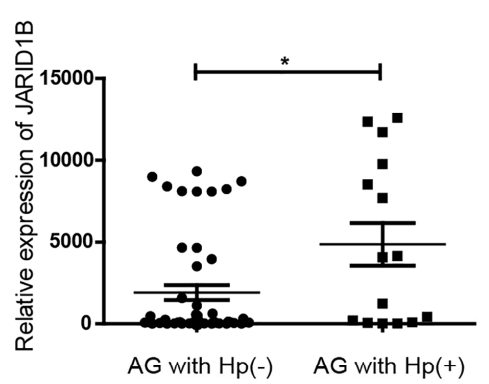

B

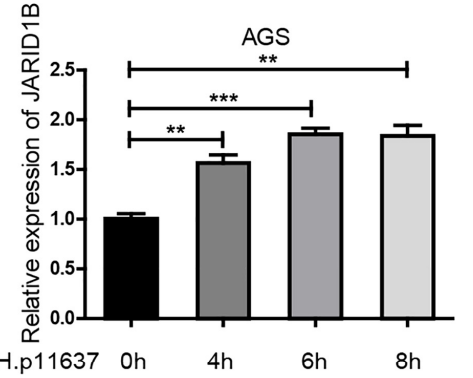

c

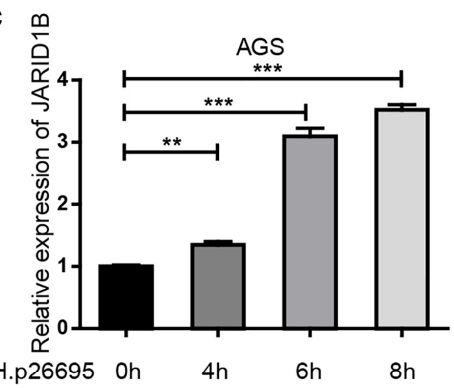

$\mathbf{F}$

D $\stackrel{m}{\frac{9}{2}}$

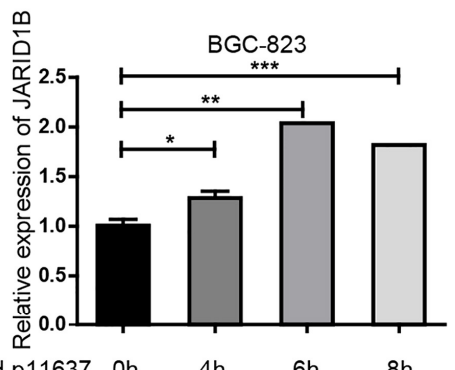

E

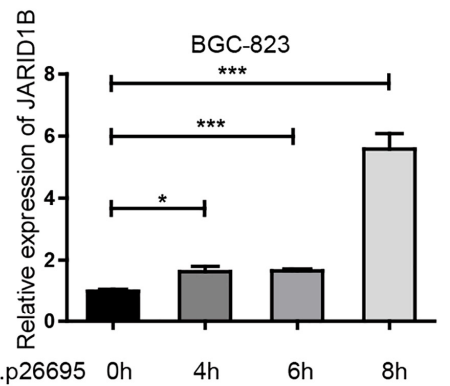

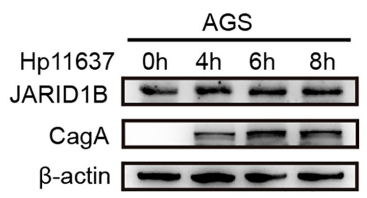

G
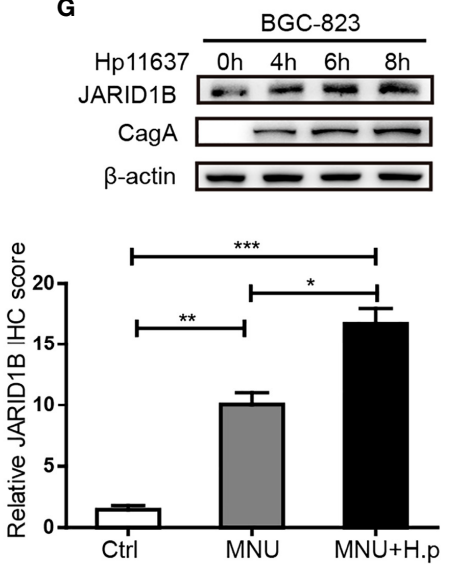

FIGURE 4 | H. pylori promotes the expression of JARID1B in gastric cancer (GC). (A) JARID1B expression was analyzed in gastritis tissue with $H$. pylori positive or negative by RT-qPCR. (B-E) Real-time quantitative PCR (RT-GPCR) was used to detect JARID1B messenger RNA (mRNA) in AGS and BGC-823 cell lines treated with different strains of $H$. pylori at different time points, ${ }^{\star} \mathrm{P}<0.05$, ${ }^{\star *} \mathrm{P}<0.01,{ }^{\star \star \star} \mathrm{P}<0.001$ by Student's t-test. (F, G) Western blot was used to detect the protein levels of JARID1B in AGS and BGC-823 cells incubated with H. pylori strains (11637) at different time points. (H) Immunohistochemical staining analysis of JARID1B expression in gastric tissue of control group, low-dose MNU gavage group, and $H$. pylori (SSI) combined with low-dose MNU gavage group. Representative images are shown here (magnification: $\times 100, \times 400$; scale bars: 200 and $50 \mu \mathrm{m}$ ).

(Figure 5K). In addition, H. pylori infection promoted the expression of JARID1B in AGS and BGC-823 cells, while JARID1B expression was downregulated when cells were transfected with miR-29c analogs (Figures 5L, M). These results indicate that $H$. pylori regulates the expression of JARID1B through miR-29c.

\section{H. pylori Promotes Proliferation of Gastric Cancer Cells Through JARID1B}

To gain a deeper understanding of the effects of JARID1B, we performed gene set enrichment analysis (GSEA) on the microarray data from TCGA database. This revealed that high
JARID1B expression was positively associated with the G2M checkpoint and cell cycle control in GC (Figures 6A, B). As JARID1B can promote the proliferation of GC cells (Figure S5), to further explore the role of JARID1B in the proliferation induced by $H$. pylori, we performed colony formation and EdU staining experiments. We showed that the colony-forming capacity of GC cells in which JARID1B expression was inhibited was decreased relative to controls and that $H$. pylori infection increased colony formation. Furthermore, H. pylori infection together with JARID1B interference decreased colony formation relative to cells only infected with $H$. pylori (Figures 6C, D). The results of the EdU staining experiment were consistent with the colony 


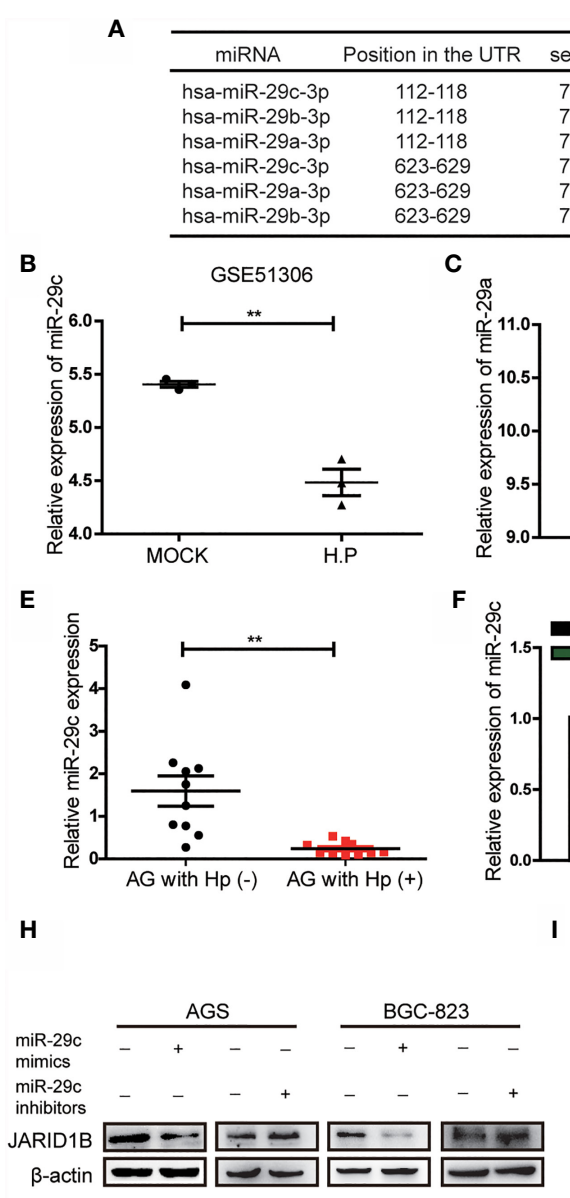

\begin{tabular}{cccccc}
\hline miRNA & Position in the UTR & seed match & context++ score & context++ score percentile & Pct \\
\hline hsa-miR-29c-3p & $112-118$ & $7 m e r-1 A$ & -0.25 & 88 & 0.95 \\
hsa-miR-29b-3p & $112-118$ & $7 m e r-1 A$ & -0.25 & 88 & 0.95 \\
hsa-miR-29a-3p & $112-118$ & $7 m e r-1 A$ & -0.24 & 87 & 0.95 \\
hsa-miR-29c-3p & $623-629$ & $7 m e r-m 8$ & -0.22 & 85 & 0.87 \\
hsa-miR-29a-3p & $623-629$ & $7 m e r-m 8$ & -0.22 & 85 & 0.87 \\
hsa-miR-29b-3p & $623-629$ & $7 m e r-m 8$ & -0.22 & 84 & 0.87 \\
\hline
\end{tabular}

H

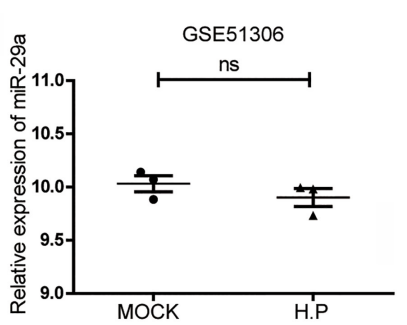

D

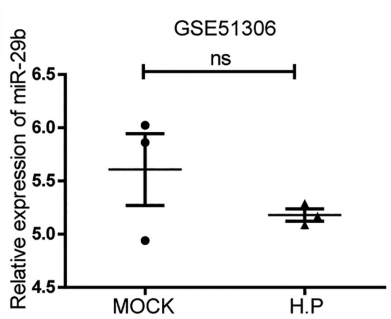

G

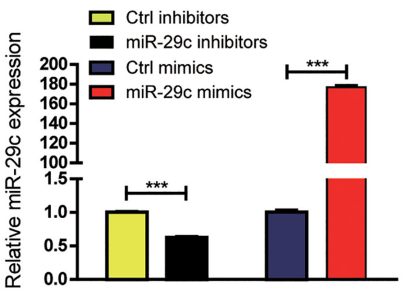

K $\square$ Ctrl mimics+JARID1B-WT-3'UTR

$\square$ miR-29c mimics+JARID1B-WT-3'UTR

- miR-29c mimics+JARID1B-Mut-3'UTR
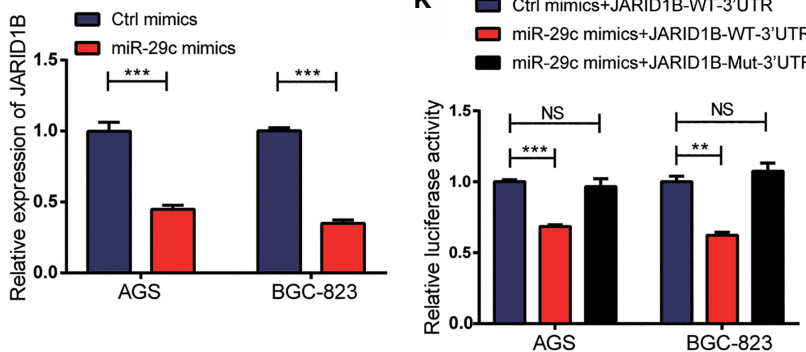

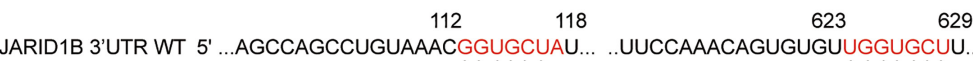

|| ||||

miR-29c 3' AUUGGCUAAAGUUUACCACGAU

AUUGGCUAAAGUUUACCACG

JARID1B 3'UTR Mut 5' ...AGCCAGCCUGUAAACTTGTTGGU.

. UUCCAAACAGUGUGUCAACAAGU..

$\mathbf{L}$
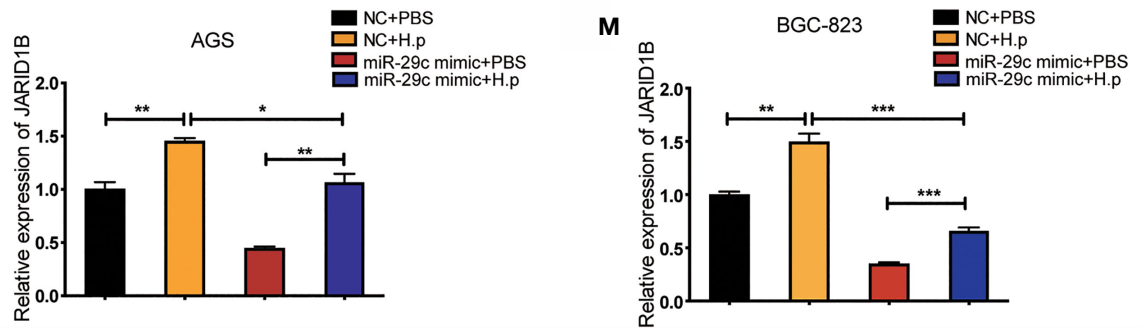

FIGURE 5 | H. pylori upregulates JARID1B expression through the downregulation of miR-29c. (A) TargetScan analysis of the microRNA that can bind to 3'untranslated region (UTR) of JARID1B. (B-D) The expression of miR-29c, miR-29a, and miR-29b in gastric epithelial cells infected with H. pylori, data came from GSE51306. (E) RT-PCR detection of miR-29c in gastritis tissues with or without $H$. pylori infection. (F) RT-PCR analysis of miR-29c expression in H. pylori-infected BGC-823 and AGS. (G) Transfection efficiency of transfected miR-29c inhibitors and mimics in GC cells AGS. (H) Western blot assay was performed to detect the expression of JARID1B protein in AGS and BGC-823 cells after transfection of miR-29c mimics and miR-29c inhibitor, respectively. (I) RT-PCR was used to detect the expression of JARID1B messenger RNA (mRNA) after transfection of miR-29c mimics in AGS and BGC-823. (J) The construction of the plasmid with miR-29c binding site [JARID1B-3'UTR-wild type (WT)] or the binding site mutation plasmid (JARID1B-3'-UTR-Mut). (K) Dual luciferase assay was performed to detect AGS and BGC-823 transfected with the miR-29c mimics and then transfected with the WT JARID1B 3'-UTR reporter plasmid or the mutant JARID1B 3'-UTR Mut reporter plasmid. (L, M) RT-PCR was used to detect JARID1B expression in AGS and BGC-823 of the control group, $H$. pylori infection group, and $H$. pylori infection combined with transfection of the miR-29c mimics group. All results were repeated three times. ${ }^{*} P<0.05$; ${ }^{* \star} P<0.01$; ${ }^{* \star} P<0.001$, NS, not statistically significant (Student's t-test). 
A

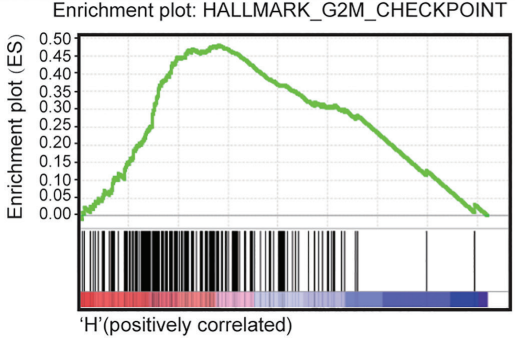

C

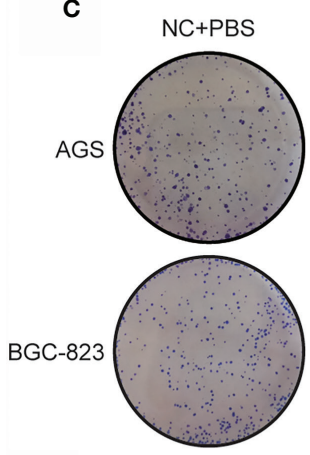

E

NC+PBS
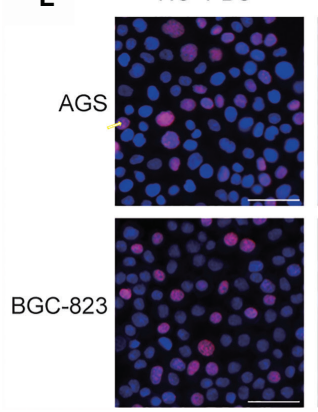
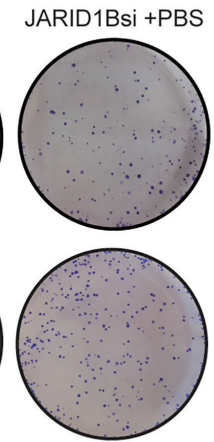

JARID1Bsi +PBS
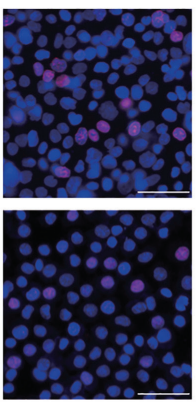

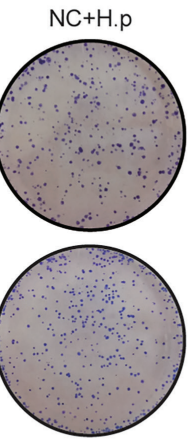

$\mathrm{NC}+\mathrm{H} \cdot \mathrm{p}$

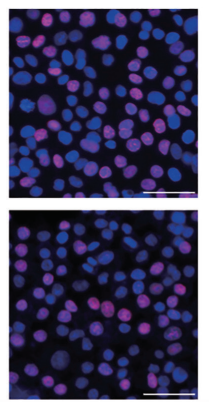

B
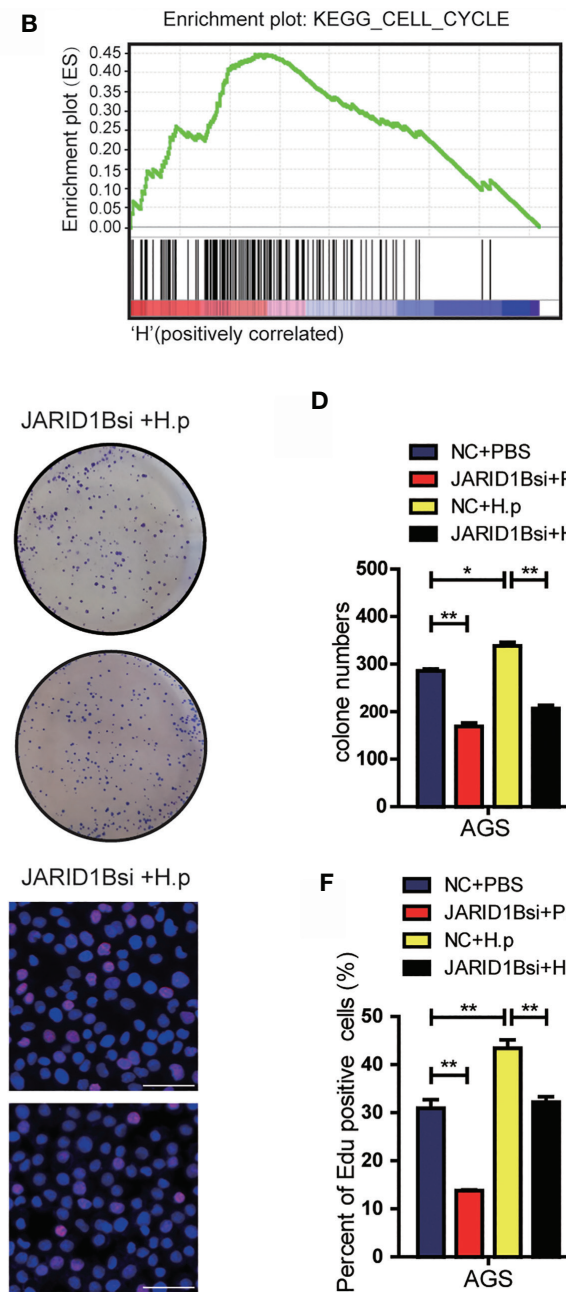

D
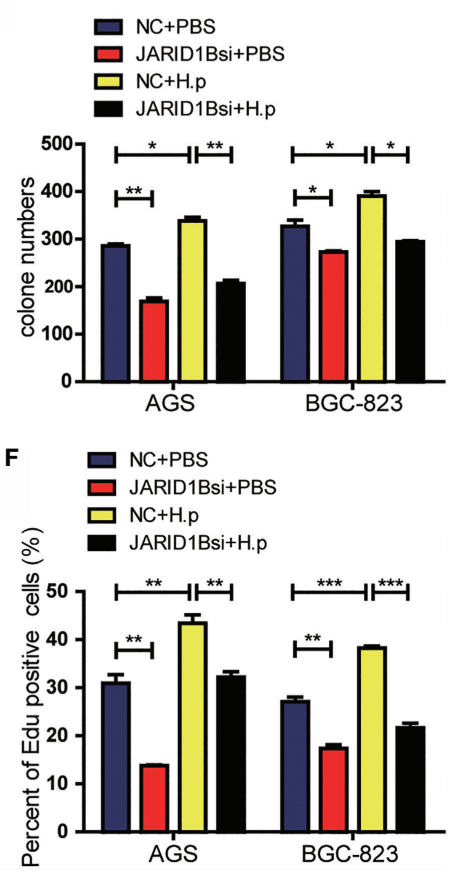

FIGURE 6 | H. pylori promotes the proliferation of gastric cancer (GC) cells via JARID1B. (A, B) Enrichment plots from gene set enrichment analysis (GSEA). (C, D) Cloning formation test including the control group, JARID1B siRNA group, $H$. pylori infection group, and JARID1B siRNA combined with $H$. pylori infection group in AGS and BGC-823 cells, representative picture (C) and quantitative map (D). (E, F) EdU assay including the control group, JARID1B siRNA group, H. pylori infection group, and JARID1B siRNA combined with $H$. pylori infection group in AGS and BGC-823, representative picture (E) and quantified map (F). All results were repeated three times, and each horizontal line in the quantified graph of the figure represents the mean \pm standard error of three experiments. Scale bar, $50 \mu m$. ${ }^{\star} \mathrm{P}<0.05 ;{ }^{\star \star} \mathrm{P}<0.01 ;{ }^{\star \star *} \mathrm{P}<0.001$ (Student's t-test).

formation findings (Figures 6E, F). Our data thus indicate that JARID1B promotes the proliferation of GC cells and is a node molecule for GC cell proliferation induced by $H$. pylori.

\section{JARID1B Promotes the Expression of CCND1 in Gastric Cancer Cells}

Finally, we aimed to elucidate the mechanisms by which JARID1B promotes GC cell proliferation. In this study, we analyzed the GSE54129 dataset in the GEO database. A search for upregulated DEGs positively associated with JARID1B was performed with Cytohubba. This showed that a number of genes are related to JARID1B expression, with CCND1 being the strongest (Figure 7A). Biological function and pathway enrichment analyses with Metascape indicated effects on six biological processes, most significantly was cancer pathways
(Figure 7B). The analysis of 45 pairs of GC and corresponding adjacent normal tissues in the GSE63089 also revealed a significant positive correlation between JARID1B and CCND1 (Figure 7C). Furthermore, the expression levels of JARID1B and several proliferation-associated genes including cyclins (CCND1, CCND2, CCND3, CCNB1, and CCNE1) and cell cycle proteindependent kinases (CDK1, CDK2, CDK3, CDK4, and CDK7) were analyzed. We observed that silencing of JARID1B decreased the expression of CCND1 in AGS and BGC-823 cells, both by RT-qPCR and Western blotting (Figures 7D-F and S6). Because JARID1B was reported to decrease H3K27me3 levels in osteosarcoma (29), we then tested the change of H3K27me3 in GC cells. The results showed that knockdown of JARID1B could enhance $\mathrm{H} 3 \mathrm{~K} 27 \mathrm{me} 3$ levels in AGS and BGC-823 cells (Figures $\mathbf{7 G}$ and $\mathbf{S 7}, \mathbf{8}$ ). In addition, as $\mathrm{H} 3 \mathrm{~K} 27 \mathrm{me} 3$ is 
A

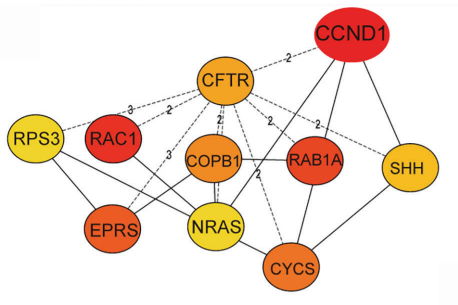

C

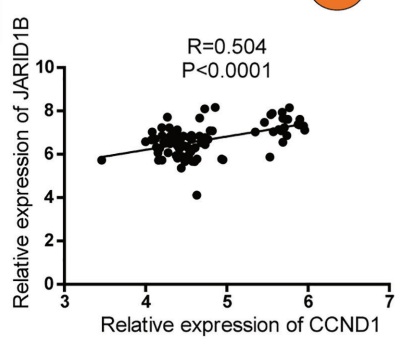

B

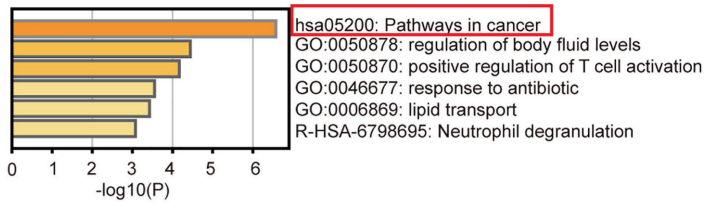

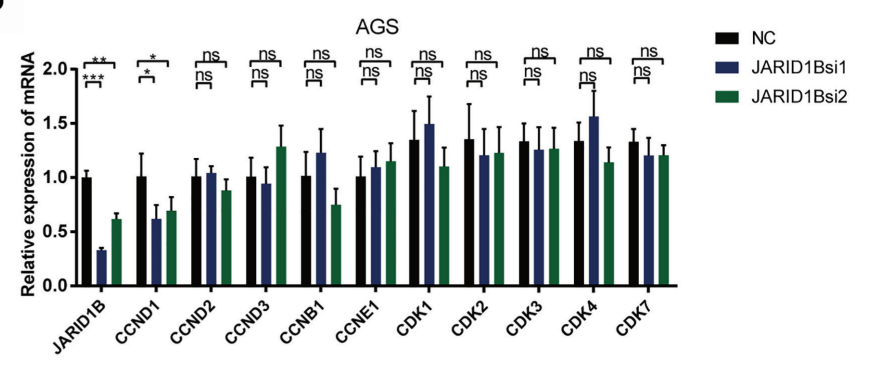

E

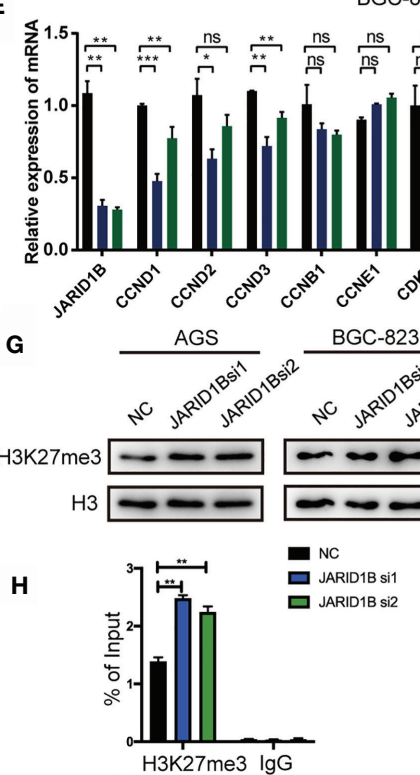

BGC-823
- NC

- JARID1Bsi1

- JARID1Bsi2

$$
\mathbf{F}
$$$$
\mathbf{F}
$$
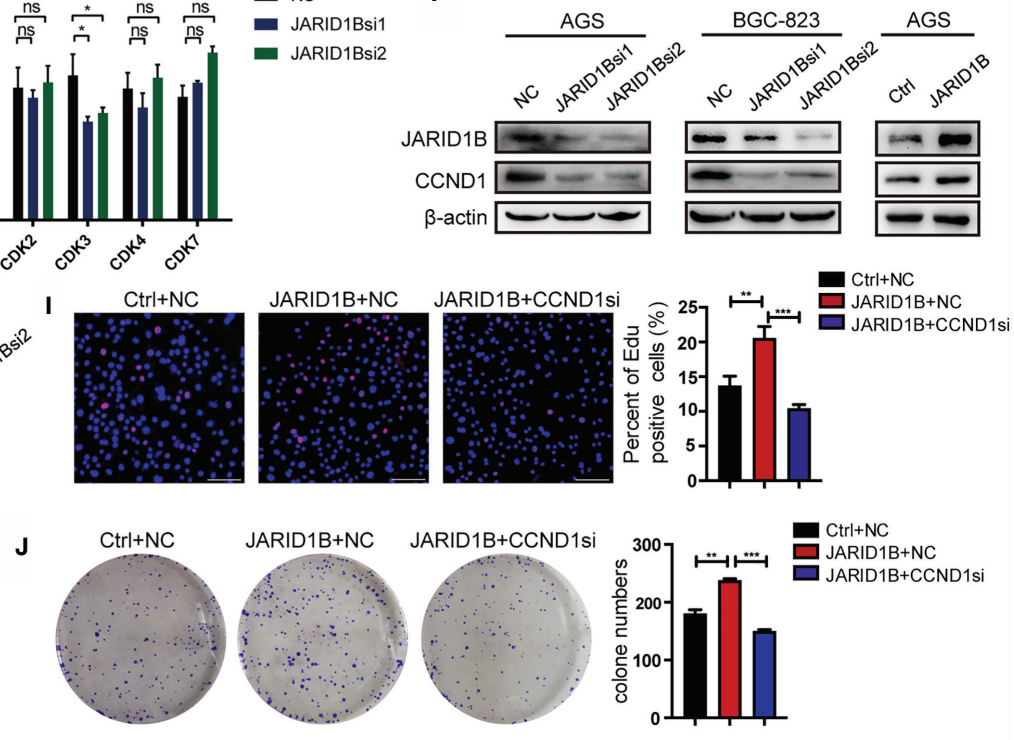

FIGURE 7 | JARID1B promotes the expression of CCND1 in gastric cancer (GC) cells. (A) The upregulated genes that are positively related to JARID1B expression in GSE54129 of GC. (B) The biological function and pathway enrichment analysis with the Metascape. (C) The Gene Expression Omnibus (GEO) database (GSE63089) analysis showed that there was a significant positive correlation between JARID1B and CCND1 in 45 pairs of GC and corresponding adjacent normal tissues. (D, E) RT-PCR was used to detect the expression of proliferation-related genes in AGS and BGC-823 transfected with JARID1B siRNA. ${ }^{*} P<0.05$; ${ }^{* *} P<$ 0.01; ${ }^{\star \star \star} \mathrm{P}<0.001$, NS, not statistically significant. (Student's t-test). (F) Western blot assay was performed to detect CCND1 expression in AGS and BGC-823 after transfection with JARID1B siRNA. (G) The levels of H3K27me3 after JARID1B knockdown was tested by Western blot. (H) Chromatin immunoprecipitation (ChIP) assays were performed in JARID1B knockdown AGS cells. (I, J) Colony formation assays and EdU staining were used to test the role of CCND1 in the proliferation of GC cells regulated by JARID1B. Scale bar, $100 \mu \mathrm{m}$.

associated with transcriptional repression (30), we further tested the correlation between JARID1B expression and H3K27me3 levels at the promoter of CCND1. ChIP results indicated that knockdown of JARID1B could enhance H3K27me3 levels in AGS cells (Figure $\mathbf{7 H}$ ). Furthermore, the results of colony formation assays and EdU staining also showed that CCND1 is a node molecule in the proliferation of GC cells regulated by JARID1B (Figures 7I, J). Therefore, our data indicate that JARID1B can regulate CCND1 expression and thereby mediate the proliferation of GC cells.

\section{DISCUSSION}

The role of histone demethylases in tumorigenesis and progression has become more clearly recognized recently, for example, KDM4B is involved in the growth and progression of tumors such as GC, breast cancer, and ovarian cancer (31-33); HPV16 E7 and miR-342-3p stimulate the expression of KDM2A in tumors, and KDM2A then enhances ERK1/2-mediated tumor formation in lung cancer (34-36); KDM4A is abnormally expressed in colorectal cancer, prostate cancer, lung cancer, 
and breast cancer and promotes their progression $(37,38)$. It has also been reported that JARID1B is overexpressed in GC and promotes cell proliferation and metastasis (20). Here, we mainly focused on the mechanism of JARID1B upregulation in GC and report its vital role for the clinical prognosis.

Chronic H. pylori infection is an important risk factor for developing gastric adenocarcinoma (3). It has been reported that $H$. pylori promotes the release of inflammatory factors and the proliferation of epithelial cells by activating phosphoinositide 3kinase (PI3K)/Akt, Wnt/ $\beta$-catenin, nuclear factor (NF)- $\kappa \mathrm{B}$, and Ras signaling pathways (39-43). H. pylori-mediated NF- $\kappa \mathrm{B} / \mathrm{miR}-$ 223-3p/ARID1A axis signaling could promote the development and progression of GC (44). Our group has demonstrated that H. pylori induces hepatocyte nuclear factor-4 $\alpha(\mathrm{HNF} 4 \alpha)$ expression via the NF- $\kappa \mathrm{B}$ pathway (45). In the present study, analyses of clinical specimens showed that JARID1B expression was upregulated in $H$. pylori-positive gastritis tissues. Moreover, both in vitro and in vivo studies indicated that $H$. pylori infection can induce the expression of JARID1B.

miRNAs are important posttranscriptional regulators, and $H$. pylori infection may contribute to their dysregulation (46-48). Here, we found that $H$. pylori infection inhibited miR-29c expression and led to the enhancement of JARID1B by targeting its $3^{\prime}$-UTR region in gastric epithelial cells. In addition to the posttranscriptional regulation, transcriptional regulation also plays important roles in gene expression control. Our analysis of the JARID1B promoter region showed that transcription factors E2F1, p53, Sp1, and Sp3 are also potential regulators of JARID1B expression. Therefore, we will further explore the regulatory effect of these transcription factors on JARID1B expression and their specific mechanisms in the future.

Sustained cell proliferation is one of the hallmarks of tumorigenesis (49). Abnormal proliferation of cells is subject to complex regulatory processes accompanied by disordered expression of oncogenes or cell cycle-related genes. Many abnormally expressed genes mediate tumor development and progression by regulating cell proliferation. For example, SMYD3 promotes tumorigenicity and intrahepatic metastasis of hepatocellular carcinoma cells by regulating CDK2 and matrix metalloproteinase (MMP)2 expression (50). Both our colony formation and EDU experiments showed that JARID1B interference significantly inhibits the proliferation of GC cells induced by $H$. pylori infection. Moreover, our further analyses indicated that JARID1B downregulation prevents the expression of CCND1. Because CCND1 is a cell cycle regulatory protein that influences cell proliferation and has cancer-promoting properties in many tumors including GC (51), JARID1B may promote cell proliferation through CCND1. On the other hand, we also found that JARID1B may promote the expression of CCND1 through the regulation of $\mathrm{H} 3 \mathrm{~K} 27 \mathrm{me} 3$ levels at the promoter of CCND1.

In summary, this study explored the mechanisms responsible for upregulating the histone demethylase JARID1B in GC. We first revealed that $H$. pylori infection can promote JARID1B expression in GC cells through miR-29c. High expression of JARID1B in GC patients indicates a poorer prognosis, and interference of JARID1B may attenuate the proliferation of GC cells induced by $H$. pylori. This study further contributes to our understanding of gene regulation abnormalities in the development of GC and has important implications for intervention to prevent malignant transformation induced by H. pylori infection.

\section{DATA AVAILABILITY STATEMENT}

The original contributions presented in the study are included in the article/Supplementary Material. Further inquiries can be directed to the corresponding author.

\section{ETHICS STATEMENT}

The studies involving human participants were reviewed and approved by Shandong University Research Ethics Committee. The patients/participants provided their written informed consent to participate in this study. The animal study was reviewed and approved by Shandong University Research Ethics Committee.

\section{AUTHOR CONTRIBUTIONS}

JJ designed the study. LZ, YWu, LS, XL, SL, TL, WJS, WS, and YWa performed experiments. LZ, ZY, and FL analyzed data. JJ, LZ, and LM obtained funding. LZ and JJ prepared the figures. LZ and JJ wrote the manuscript. JJ supervised the study. All authors contributed to the article and approved the submitted version.

\section{FUNDING}

This work was supported by the National Natural Science Foundation of China (Nos. 81772151, 81971901, 82002107, 81871620,82172284 and 81801983) and the Department of Science and Technology of Shandong Province (No. 2018CXGC1208).

\section{ACKNOWLEDGMENTS}

We thank the patients and the investigators who participated in this study.

\section{SUPPLEMENTARY MATERIAL}

The Supplementary Material for this article can be found online at: https://www.frontiersin.org/articles/10.3389/fonc.2021.757497/ full\#supplementary-material 


\section{REFERENCES}

1. Zhang XY, Zhang PY. Gastric Cancer: Somatic Genetics as a Guide to Therapy. $J$ Med Genet (2017) 54(5):305-12. doi: 10.1136/jmedgenet-2016-104171

2. Hohenberger P, Gretschel S. Gastric Cancer. Lancet (2003) 362(9380):305-15. doi: 10.1016/s0140-6736(03)13975-x

3. Amieva M, Peek RJ. Pathobiology of Helicobacter Pylori-Induced Gastric Cancer. Gastroenterology (2016) 150(1):64-78. doi: 10.1053/j.gastro.2015.09.004

4. Choi IJ, Kook MC, Kim YI, Cho SJ, Lee JY, Kim CG, et al. Helicobacter Pylori Therapy for the Prevention of Metachronous Gastric Cancer. N Engl J Med (2018) 378(12):1085-95. doi: 10.1056/NEJMoa1708423

5. Maeda S, Ogura K, Yoshida H, Kanai F, Ikenoue T, Kato N, et al. Major Virulence Factors, VacA and CagA, Are Commonly Positive in Helicobacter Pylori Isolates in Japan. Gut (1998) 42(3):338-43. doi: 10.1136/gut.42.3.338

6. Graham DY. History of Helicobacter Pylori, Duodenal Ulcer, Gastric Ulcer and Gastric Cancer. World J Gastroenterol (2014) 20(18):5191-204. doi: 10.3748/wjg.v20.i18.5191

7. Hanada K, Graham DY. Helicobacter Pylori and the Molecular Pathogenesis of Intestinal-Type Gastric Carcinoma. Expert Rev Anticancer Ther (2014) 14 (8):947-54. doi: 10.1586/14737140.2014.911092

8. Shiotani A, Cen P, Graham DY. Eradication of Gastric Cancer Is Now Both Possible and Practical. Semin Cancer Biol (2013) 23(6 Pt B):492-501. doi: 10.1016/j.semcancer.2013.07.004

9. Hojfeldt JW, Agger K, Helin K. Histone Lysine Demethylases as Targets for Anticancer Therapy. Nat Rev Drug Discovery (2013) 12(12):917-30. doi: $10.1038 / \operatorname{nrd} 4154$

10. Majello B, Gorini F, Sacca CD, Amente S. Expanding the Role of the Histone Lysine-Specific Demethylase LSD1 in Cancer. Cancers (Basel) (2019) 11 (3):324. doi: 10.3390/cancers11030324

11. Hayami S, Kelly JD, Cho HS, Yoshimatsu M, Unoki M, Tsunoda T, et al. Overexpression of LSD1 Contributes to Human Carcinogenesis Through Chromatin Regulation in Various Cancers. Int J Cancer (2011) 128(3):57486. doi: 10.1002/ijc.25349

12. Lv T, Yuan D, Miao X, Lv Y, Zhan P, Shen X, et al. Over-Expression of LSD1 Promotes Proliferation, Migration and Invasion in Non-Small Cell Lung Cancer. PloS One (2012) 7(4):e35065. doi: 10.1371/journal.pone.0035065

13. Han F, Ren J, Zhang J, Sun Y, Ma F, Liu Z, et al. JMJD2B Is Required for Helicobacter Pylori-Induced Gastric Carcinogenesis. Via Regulating COX-2 Expression. Oncotarget (2016) 7(25):38626-37. doi: 10.18632/oncotarget.9573

14. Zhao L, Li W, Zang W, Liu Z, Xu X, Yu H, et al. JMJD2B Promotes EpithelialMesenchymal Transition by Cooperating With Beta-Catenin and Enhances Gastric Cancer Metastasis. Clin Cancer Res (2013) 19(23):6419-29. doi: 10.1158/1078-0432.CCR-13-0254

15. Zhang Y, Yang H, Guo X, Rong N, Song Y, Xu Y, et al. The PHD1 Finger of KDM5B Recognizes Unmodified H3K4 During the Demethylation of Histone H3K4me2/3 by KDM5B. Protein Cell (2014) 5(11):837-50. doi: 10.1007/ s13238-014-0078-4

16. Yamamoto S, Wu Z, Russnes HG, Takagi S, Peluffo G, Vaske C, et al. JARID1B Is a Luminal Lineage-Driving Oncogene in Breast Cancer. Cancer Cell (2014) 25(6):762-77. doi: 10.1016/j.ccr.2014.04.024

17. Yamane K, Tateishi K, Klose RJ, Fang J, Fabrizio LA, Erdjument-Bromage H, et al. PLU-1 Is an H3K4 Demethylase Involved in Transcriptional Repression and Breast Cancer Cell Proliferation. Mol Cell (2007) 25(6):801-12. doi: 10.1016/j.molcel.2007.03.001

18. Hayami S, Yoshimatsu M, Veerakumarasivam A, Unoki M, Iwai Y, Tsunoda $\mathrm{T}$, et al. Overexpression of the JmjC Histone Demethylase KDM5B in Human Carcinogenesis: Involvement in the Proliferation of Cancer Cells Through the E2F/RB Pathway. Mol Cancer (2010) 9:59. doi: 10.1186/1476-4598-9-59

19. Xiang Y, Zhu Z, Han G, Ye X, Xu B, Peng Z, et al. JARID1B Is a Histone H3 Lysine 4 Demethylase Up-Regulated in Prostate Cancer. Proc Natl Acad Sci USA (2007) 104(49):19226-31. doi: 10.1073/pnas.0700735104

20. Wang Z, Tang F, Qi G, Yuan S, Zhang G, Tang B, et al. KDM5B Is Overexpressed in Gastric Cancer and Is Required for Gastric Cancer Cell Proliferation and Metastasis. Am J Cancer Res (2015) 5(1):87-100.

21. Liang X, Zeng J, Wang L, Shen L, Li S, Ma L, et al. Histone Demethylase RBP2 Induced by Helicobactor Pylori CagA Participates in the Malignant Transformation of Gastric Epithelial Cells. Oncotarget (2014) 5(14):5798-807. doi: 10.18632/oncotarget.2185
22. Liang X, Zeng J, Wang L, Shen L, Ma X, Li S, et al. Histone Demethylase RBP2 Promotes Malignant Progression of Gastric Cancer Through TGF-Beta1(P-Smad3)-RBP2-E-Cadherin-Smad3 Feedback Circuit. Oncotarget (2015) 6 (19):17661-74. doi: 10.18632/oncotarget.3756

23. Li S, Sun A, Liang X, Ma L, Shen L, Li T, et al. Histone Demethylase PHF8 Promotes Progression and Metastasis of Gastric Cancer. Am J Cancer Res (2017) 7(3):448-61. doi: 10.1158/0008-5472.CAN-16-2350

24. Chin $\mathrm{CH}$, Chen SH, Wu HH, Ho CW, Ko MT, Lin CY. Cytohubba: Identifying Hub Objects and Sub-Networks From Complex Interactome. BMC Syst Biol (2014) 8(Suppl 4):S11. doi: 10.1186/1752-0509-8-S4-S11

25. Tripathi S, Pohl MO, Zhou Y, Rodriguez-Frandsen A, Wang G, Stein DA, et al. Meta- and Orthogonal Integration of Influenza "OMICs" Data Defines a Role for UBR4 in Virus Budding. Cell Host Microbe (2015) 18(6):723-35. doi: 10.1016/j.chom.2015.11.002

26. Nam KT, Hahm KB, Oh SY, Yeo M, Han SU, Ahn B, et al. The Selective Cyclooxygenase-2 Inhibitor Nimesulide Prevents Helicobacter PyloriAssociated Gastric Cancer Development in a Mouse Model. Clin Cancer Res (2004) 10(23):8105-13. doi: 10.1158/1078-0432.CCR-04-0896

27. Link A, Kupcinskas J. MicroRNAs as non-Invasive Diagnostic Biomarkers for Gastric Cancer: Current Insights and Future Perspectives. World J Gastroenterol (2018) 24(30):3313-29. doi: 10.3748/wjg.v24.i30.3313

28. Chmiela M, Kupcinskas J. Review: Pathogenesis of Helicobacter Pylori Infection. Helicobacter (2019) 24(Suppl 1):e12638. doi: 10.1111/hel.12638

29. Wang W, Zheng K, Pei Y, Zhang X. Histone Demethylase JARID1B Is Overexpressed in Osteosarcoma and Upregulates Cyclin D1 Expression via Demethylation of H3k27me3. Oncol Res (2018) 26(3):373-84. doi: 10.3727/ $096504017 X 14939809845080$

30. Zhang YZ, Yuan J, Zhang L, Chen C, Wang Y, Zhang G, et al. Coupling of H3K27me3 Recognition With Transcriptional Repression Through the BAHPHD-CPL2 Complex in Arabidopsis. Nat Commun (2020) 11(1):6212. doi: 10.1038/s41467-020-20089-0

31. Wu MC, Cheng HH, Yeh TS, Li YC, Chen TJ, Sit WY, et al. KDM4B Is a Coactivator of C-Jun and Involved in Gastric Carcinogenesis. Cell Death Dis (2019) 10(2):68. doi: 10.1038/s41419-019-1305-y

32. Johmura Y, Maeda I, Suzuki N, Wu W, Goda A, Morita M, et al. Fbxo22Mediated KDM4B Degradation Determines Selective Estrogen Receptor Modulator Activity in Breast Cancer. J Clin Invest (2018) 128(12):5603-19. doi: $10.1172 / J C I 121679$

33. Wilson C, Qiu L, Hong Y, Karnik T, Tadros G, Mau B, et al. The Histone Demethylase KDM4B Regulates Peritoneal Seeding of Ovarian Cancer. Oncogene (2017) 36(18):2565-76. doi: 10.1038/onc.2016.412

34. Wang F, Liang S, Liu X, Han L, Wang J, Du Q. LINC00460 Modulates KDM2A to Promote Cell Proliferation and Migration by Targeting miR-3423p in Gastric Cancer. Onco Targets Ther (2018) 11:6383-94. doi: 10.2147/ OTT.S169307

35. Ou R, Zhu L, Zhao L, Li W, Tao F, Lu Y, et al. HPV16 E7-Induced Upregulation of KDM2A Promotes Cervical Cancer Progression by Regulating miR-132-Radixin Pathway. J Cell Physiol (2019) 234(3):2659-71. doi: $10.1002 /$ jcp. 27080

36. Wagner KW, Alam H, Dhar SS, Giri U, Li N, Wei Y, et al. KDM2A Promotes Lung Tumorigenesis by Epigenetically Enhancing ERK1/2 Signaling. J Clin Invest (2013) 123(12):5231-46. doi: 10.1172/JCI68642

37. Kim TD, Shin S, Berry WL, Oh S, Janknecht R. The JMJD2A Demethylase Regulates Apoptosis and Proliferation in Colon Cancer Cells. J Cell Biochem (2012) 113(4):1368-76. doi: 10.1002/jcb.24009

38. Berry WL, Shin S, Lightfoot SA, Janknecht R. Oncogenic Features of the JMJD2A Histone Demethylase in Breast Cancer. Int J Oncol (2012) 41 (5):1701-6. doi: 10.3892/ijo.2012.1618

39. Wen J, Wang Y, Gao C, Zhang G, You Q, Zhang W, et al. Helicobacter Pylori Infection Promotes Aquaporin 3 Expression via the ROS-HIF-1alpha-AQP3ROS Loop in Stomach Mucosa: A Potential Novel Mechanism for Cancer Pathogenesis. Oncogene (2018) 37(26):3549-61. doi: 10.1038/s41388-0\180208-1

40. Suzuki M, Mimuro H, Kiga K, Fukumatsu M, Ishijima N, Morikawa H, et al. Helicobacter Pylori CagA Phosphorylation-Independent Function in Epithelial Proliferation and Inflammation. Cell Host Microbe (2009) 5 (1):23-34. doi: 10.1016/j.chom.2008.11.010 
41. Murata-Kamiya N, Kurashima Y, Teishikata Y, Yamahashi Y, Saito Y, Higashi $\mathrm{H}$, et al. Helicobacter Pylori CagA Interacts With E-Cadherin and Deregulates the Beta-Catenin Signal That Promotes Intestinal Transdifferentiation in Gastric Epithelial Cells. Oncogene (2007) 26(32):4617- 26. doi: 10.1038/sj.onc.1210251

42. Zeaiter Z, Cohen D, Musch A, Bagnoli F, Covacci A, Stein M. Analysis of Detergent-Resistant Membranes of Helicobacter Pylori Infected Gastric Adenocarcinoma Cells Reveals a Role for MARK2/Parlb in CagA-Mediated Disruption of Cellular Polarity. Cell Microbiol (2008) 10(3):781-94. doi: 10.1111/j.1462-5822.2007.01084.x

43. Nusse R, Clevers H. Wnt/beta-Catenin Signaling, Disease, and Emerging Therapeutic Modalities. Cell (2017) 169(6):985-99. doi: 10.1016/j.cell.2017.05.016

44. Yang F, Xu Y, Liu C, Ma C, Zou S, Xu X, et al. NF-Kappab/miR-223-3p/ ARID1A Axis Is Involved in Helicobacter Pylori CagA-Induced Gastric Carcinogenesis and Progression. Cell Death Dis (2018) 9(1):12. doi: 10.1038/s41419-017-0020-9

45. Ma L, Zeng J, Guo Q, Liang X, Shen L, Li S, et al. Mutual Amplification of HNF4alpha and IL-1R1 Composes an Inflammatory Circuit in Helicobacter Pylori Associated Gastric Carcinogenesis. Oncotarget (2016) 7(10):11349-63. doi: 10.18632/oncotarget.7239

46. Iorio MV, Croce CM. MicroRNAs in Cancer: Small Molecules With a Huge Impact. J Clin Oncol (2009) 27(34):5848-56. doi: 10.1200/JCO.2009.24.0317

47. Geng Y, Lu X, Wu X, Xue L, Wang X, Xu J. MicroRNA-27b Suppresses Helicobacter Pylori-Induced Gastric Tumorigenesis Through Negatively Regulating Frizzled7. Oncol Rep (2016) 35(4):2441-50. doi: 10.3892/ or.2016.4572

48. Li S, Liang X, Ma L, Shen L, Li T, Zheng L, et al. MiR-22 Sustains NLRP3 Expression and Attenuates H. Pylori-Induced Gastric Carcinogenesis. Oncogene (2018) 37(7):884-96. doi: 10.1038/onc.2017.381
49. Hanahan D, Weinberg RA. Hallmarks of Cancer: The Next Generation. Cell (2011) 144(5):646-74. doi: 10.1016/j.cell.2011.02.013

50. Wang Y, Xie BH, Lin WH, Huang YH, Ni JY, Hu J, et al. Amplification of SMYD3 Promotes Tumorigenicity and Intrahepatic Metastasis of Hepatocellular Carcinoma via Upregulation of CDK2 and MMP2. Oncogene (2019) 38(25)4948-61. doi: 10.1038/s41388-019-0766-x

51. John RR, Malathi N, Ravindran C, Anandan S. Mini Review: Multifaceted Role Played by Cyclin D1 in Tumor Behavior. Indian J Dent Res (2017) 28 (2):187-92. doi: 10.4103/ijdr.IJDR_697_16

Conflict of Interest: The authors declare that the research was conducted in the absence of any commercial or financial relationships that could be construed as a potential conflict of interest.

Publisher's Note: All claims expressed in this article are solely those of the authors and do not necessarily represent those of their affiliated organizations, or those of the publisher, the editors and the reviewers. Any product that may be evaluated in this article, or claim that may be made by its manufacturer, is not guaranteed or endorsed by the publisher.

Copyright (c) 2021 Zheng, Wu, Shen, Liang, Yang, Li, Li, Shang, Shao, Wang, Liu, Ma and Jia. This is an open-access article distributed under the terms of the Creative Commons Attribution License (CC BY). The use, distribution or reproduction in other forums is permitted, provided the original author(s) and the copyright owner(s) are credited and that the original publication in this journal is cited, in accordance with accepted academic practice. No use, distribution or reproduction is permitted which does not comply with these terms. 